\title{
TITLE:
}

\section{Particulate pattern formation and its morphology control by convective self-assembly}

$\operatorname{AUTHOR}(S)$ :

Watanabe, Satoshi; Miyahara, Minoru T.

\section{CITATION:}

Watanabe, Satoshi ...[et al]. Particulate pattern formation and its morphology control by convective self-assembly. Advanced Powder Technology 2013, 24(6): 897-907

\section{ISSUE DATE:}

2013-11

URL:

http://hdl.handle.net/2433/179776

\section{RIGHT:}

(c) 2013 The Society of Powder Technology Japan. Published by Elsevier B.V.; This is not the published version. Please cite only the published version.; この論文は出版社版でありません。引用の際には出版社版を ご確認ご利用ください。 


\title{
Particulate Pattern Formation and Its Morphology
}

\section{Control by Convective Self-Assembly}

\author{
Satoshi Watanabe and Minoru T. Miyahara* \\ Department of Chemical Engineering, Kyoto University, \\ Katsura, Nishikyo, Kyoto 615-8510, Japan
}

miyahara@cheme.kyoto-u.ac.jp

* Corresponding author 


\begin{abstract}
Bottom-up self-organization approaches are promising for fabricating higher-order patterned surfaces composed of colloidal particles. The first example among the patterns that have been extensively studied would be stripes; however, the formation of stripe patterns has so far been confined to partially or fully hydrophobic surfaces. By contrast, we have succeeded in preparing well-defined stripe patterns even on strongly hydrophilic substrates via a convective self-assembly technique. By using this technique, a stripe pattern was produced simply by suspending a substrate in a dilute suspension, without any complicated procedure; the stripes spontaneously aligned parallel to the contact line. Driven by this finding, we further investigate this self-assembly process, and find out that the convective self-assembly is quite promising as a template-free pattern formation technique. In the present paper, we first overview the convective self-assembly technique which is originally developed for uniform film formation, and then present our recent results on the pattern formation of colloidal particles through the convective self-assembly. This technique can produce various patterns including stripes, cluster arrays, and grids in response to macroscopic experimental parameters such as particle concentration and temperature.
\end{abstract}

Keywords: Self-assembly, Patterned structure, Template-free, Drying, Particulate film, Bottom-up approach, Convection 


\section{Introduction}

Significant progress has recently been made in techniques of surface patterning over large areas because micro-, meso-, and nanostructured surfaces exhibit anomalous properties due to enhanced interfacial effects [1-3]. Colloidal particles are fundamental building elements for fabricating patterned structures on a substrate including stripes, rings, grids, and dot-arrays, which can find potential applications such as controlled cell growth platform [4], transparent conductive electrodes [5, 6], and plasmonic sensing materials [7]. The fabrication of complex particulate patterns in a precisely controlled manner has been achieved by top-down lithography techniques, e.g., photolithography, laser interference lithography, and electron-beam lithography. Although these top-down approaches are quite effective, the use of bottom-up self-assembly processes becomes necessary to complement them as the required structure sizes become of the order of nanometers. The advantages of self-assembly techniques are its simplicity, versatility, cost-effectiveness, and compatibility with heterogeneous integration processes [8-10]. Among various techniques so far reported, a widely-used one is template-directed colloidal self-assembly. In this approach, a suspension droplet is allowed to evaporate on a topologically grooved substrate which acts as a template to guide and arrange the particles into the desired pattern with the aid of the convection that brings the particles to the drying front [11-13]. Instead of the topological modification, the use of chemically patterned substrates is also proposed, in which a specific interaction with particles/polymers directs them into the pre-defined pattern [14-16]. These approaches, however, require direct modification of substrate surface, and accordingly the material type of the substrate is limited. Celio et al. and Harris et al. enabled the colloidal patterning of a flat substrate without topological or chemical pre-modification by introducing a template mask set above the substrate to control solvent evaporation, thereby avoiding this problem, which they call confined dewetting lithography or evaporative lithography [17, 18]. However, this 
approach still encounters a critical problem that any desired change in pattern periodicity or morphology requires another template mask that needs to be created by costly and complicated top-down lithography techniques. In that respect, a template-free or lithography-free self-assembly technique is strongly required to realize controlled fabrication of patterned structures of colloidal particles.

As a possible candidate for the template-free fabrication, it has been demonstrated that the dewetting of a thin liquid film can yield several patterns composed of particle aggregates, such as stripes [19-21], "spoke"-like radial shapes [19], dot arrays [20, 22-24], and polygonal patterns [25], owing to the fingering instability [26] or Rayleigh instability [27]. However, the dewetting process is very sensitive to experimental conditions and accordingly poses difficulties in controlling resultant structures. Another template-free process is contact line pinning and subsequent slip motion to produce patterned structures of particles such as ring-shaped patterns on horizontally placed substrates [28-31], known as "coffee stain" patterns [32], and stripe patterns on vertically suspended surfaces in a suspension [33-35]. For the stick-and-slip motion to occur, it is said that the wettability of the substrates is quite important; partially wet substrates are favorable, while easily wettable substrates do not induce the stick-and-slip motion of the contact line [34]. With regard to the stick-and-slip motion, a critical problem in controlling the surface pattern is that the time required for the contact line to remain stuck until the next slip occurs is unavailable a priori because of its stochastic nature, leading to difficulties in predicting the periodicity of the structures. Another problem in the stick-and-slip motion is that the resultant stripes often show distorted shapes and a low degree of periodicity, although several elegant techniques recently reported have made it possible to produce well-defined patterns by successfully controlling the stick-and-slip motion in confined geometries [36-39].

The difficulty in controlling and modeling the dewetting process or stick-and-slip motion 
stems from the uncertainty of the contact line motion due to the inherent instability of the meniscus. Instead of partially wet substrates, the use of well wet substrates on which fluids spread stably can avoid the problem regarding the uncertainty of the meniscus motion, and more importantly, patterned structures can spontaneously form even on completely wetting substrates. In fact, while studying the formation of continuous particulate film by a vertical-deposition self-assembly process called the convective self-assembly method [40], we unexpectedly noticed emergence of stripe patterns on hydrophilic substrates. As a matter of fact, very few studies was succeeded in preparing pattern structures on solvophilic substrates [41]; in addition, the formation mechanism of such patterns was not clarified.

The finding of the stripe pattern formation showed us the potential of the combination of the vertical deposition process with wettable substrates as a template-free pattern formation technique, and highly motivated us to further investigate the convective self-assembly process. Through the investigation, we have revealed excellent performance of the process to spontaneously produce patterned structures of particles on flat substrates, and found out that the key factor in controlling the pattern is the behavior of the meniscus tip at which particulate films form. In this invited review paper, we first overview the convective self-assembly technique, and then introduce our recent results on pattern formation of colloidal particles by using the technique.

\section{Convective Self-Assembly}

The convective self-assembly technique is proposed by Dimitrov and Nagayama in 1996 to produce a monolayer composed of the close-packed structure of particles [40], which is based on their precedent experimental investigation on "nucleation" of a close-packed structure of particles [42]. This technique features the control of the homogeneous growth of particle arrays on the basis of a model equation derived from a mass balance in the film 
formation region. The equation is demonstrated to apply to multi-layer film formation [43]. The schematic of the experimental setup we used is shown in Fig. 1a. In this process, a well-wetted substrate is immersed vertically into a colloidal suspension and the temperature is controlled to allow the solvent to evaporate. As the liquid level descends because of evaporation, the substrate becomes dry and a particulate film appears on the substrate. A basic principle of the particle assembly is as follows. The evaporation rate around the tip of the meniscus formed on a well-wet substrate is larger than that of the bulk solvent owing to the heat transfer from the substrate. As shown in Fig. 1b, evaporation at the meniscus tip induces an upward convective flow to compensate the amount of the solvent evaporation. The convective flow then carries particles into the meniscus tip, at which the assembled particles form a close-packed particle array due to the laterally acting capillary force between the particles. By appropriately adjusting the experimental parameters such as particle concentration, humidity, and temperature as required by the operating window of parameters, this technique can produce uniform particulate films [44] and thus has been applied to various fields [45]. This technique is applicable to a wide range of particle size and type as long as the particles are stably dispersed in a solvent. As for the particle shape, not only spherical particles but also cubes [46], polyhedrons [47], nanoplates [48], nanowires [49, 50], nanotubes $[51,52]$, sheets $[53,54]$ can be assembled on a substrate. However attention should be paid to the use of particles with high density and/or large size because this technique transfers particles against gravity. In the case of the silica particle, we have confirmed that the effect of gravity is negligible for the particle size less than $500 \mathrm{~nm}$. Thus for the assembly of larger size of particles, mechanical agitation or convection flow needs to be introduced in the process to keep the particles suspended against the gravity [55-57], or another approach is to use the horizontal convective assembly [58-60].

Although the convective self-assembly by vertical deposition is originally developed to 
produce uniform particulate films, a precise control of the particle flux towards the meniscus tip can result in patterned deposits of colloidal particles because particles accumulate only at the meniscus tip, which is our prime focus of research. Following this concept, we have mainly investigated the effect of the particle concentration and temperature because the two experimental parameters directly affect the particle flux. In our experiments, the solvent was water, the substrate was glass, silicon wafer, and mica, and the particle was silica, silver, gold, and polystyrene latex (PSL). Right before use, the glass and silicon wafer substrates were treated with plasma cleaner or a 5:1:1 mixed solution of water, ammonium hydroxide $(28 \%)$, and hydrogen peroxide (30\%) [61] to clean their surface, and the mica substrate was cleaved with scotch tape, thereby obtaining fully hydrophilic surfaces. The particle assembly was conducted in a temperature-controlled incubator with the temperature from 60 to $80{ }^{\circ} \mathrm{C}$.

\section{Pattern Formation by Convective Self-Assembly}

\subsection{Stripe Pattern [62, 63]}

The resultant self-assembling structure of colloidal particles prepared by the vertical-type convective self-assembly process changes depending on the particle concentration in the suspension. In the case of the silica particle with the diameter of $120 \mathrm{~nm}$, a high concentration suspension with the volume fraction of $10^{-3} \mathrm{v} / \mathrm{v}$ typically yields a multi-layer particulate film, an example of which is shown in Fig. 2a. As shown in Fig. 2b, the film is composed of a close-packed array of particles. Note that the white lines observed in Fig. 2a are cracks formed at the drying process of the film $[64,65]$, and the prevention of their formation has been an important topic although we do not discuss here $[66,67]$. The film thickness naturally decreases with the decrease in the particle concentration because of the smaller particle flux toward the drying front. However, we find that there exists a thickness limit for the uniform film formation (e.g. 5 layers for $120 \mathrm{~nm}$ silica particles), below which no uniform films form. 
Instead, a striped pattern spontaneously forms (Figs. 2c, d). The white lines in Fig. 2c are the stripes composed of close-packed arrays of silica particles (Fig. 2d), while the gray bands form the spacing where almost no particles exist. When observed under an optical microscope, stripes exhibit brilliant colors (Fig. 2e) due to the interference of light [68]. A sharp color change corresponds to the difference in the thicknesses of the stripes (Fig. 2f), enabling us to easily identify the number of layers. The sharp color transition in the optical images of the stripes indicates that the number of layers increases stepwise with an interval of several micrometers in the upper portion of a single stripe. The stripes form parallel to the air-liquid-substrate three-phase contact line. A typical concentration range for the stripe formation is $10^{-4} \mathrm{v} / \mathrm{v}$, and we have confirmed stripe formation of several particles including silica, gold, silver, and PSL in the concentration regime. The stripe pattern thus appears to depend on the particle concentration rather than on the exact particle type. Here it should be noted that the boundary concentrations are valid only for the vertical deposition on stationary substrates, and can vary depending on experimental setups such as the vertical deposition on moving substrates [40] and the horizontal convective assembly [59].

The stripe width increases linearly with the particle concentration, as demonstrated for two different sizes of particles, and the number of layers also increases with the concentration (Fig. 3a). This is qualitatively natural because a higher particle concentration drives more particles into the meniscus tip where a particulate film is growing, resulting in a wider and thicker stripe. In contrast, the stripe spacing does not depend on the particle concentration but exhibits a mild dependence on the number of layers of the stripes (Fig. 3b). Further, we confirmed that a different descent rate at another temperature yields almost the same stripe width and spacing as far as the particle concentration remains constant. To clarify the mechanism, we directly observed the behavior of the meniscus tip during the stipe formation, the snapshots of which are shown in Fig. 4. In Fig 4a, the two pale-orange bands at the upper 
side of the figure are stripes that have already formed; the next gray band at the meniscus tip is a stripe in the process of formation, and this stripe grows in the downward direction because of the particles brought in by the convective flow. After a few moments, the meniscus tip starts to slide off from the growing stripe and exposes the substrate surface (Fig. 4b). The sliding motion of the meniscus propagates along the growing stripe from right to left, thereby terminating the stripe's growth. As a result, a new stripe forms at a regular spacing (Fig 4c). This behavior seems different from the one referred to as the pinning-depinning phenomenon which is observed experimentally [69] and described by a theoretical model [70], in which the pinned contact line jumps off from a particulate film.

The key factor to cause the sliding behavior, which we have finally found out, is that the meniscus can form a concave surface against the substrate because the meniscus is attached to the outermost particle layer that is at a certain distance from the substrate, which is in contrast to the meniscus attached to a bare substrate. The proposed mechanism is as follows. As shown in Fig. $1 \mathrm{~b}$, the meniscus tip vertically touches the tail end of the particulate film, whose width is growing due to the particle flux from underneath. Because of the present condition of a reasonably low particle concentration, the particle flux is so small that the growth rate of the particulate film becomes slower than the rate of the liquid level drop. This rate difference accordingly elongates the meniscus into a concave shape against the substrate in order to satisfy the Laplace equation in static mechanics because the formation rate is slow enough to be regarded as a quasi-static process, as shown in Fig. 5a. The concave shape of the meniscus then suppresses the particle flux to the meniscus tip because the "path" of the thin liquid film becomes narrow. The solvent evaporation further elongates and curves the meniscus, and at a certain limit, most probably the stability limit of a thin liquid film, the meniscus breaks to slide off from the particulate stripe, propagating in the lateral direction.

According to the proposed mechanism, a meniscus stuck to a thicker stripe can elongate 
further, and it accordingly offers a wider area than that obtained when the meniscus is stuck to a thinner film, which results in the dependence of the stripe spacing on the thickness of the stripe (Fig. 3b). Quantitatively, we can calculate the stripe spacing because it corresponds to the space to which particles cannot get access due to the steric hindrance of the concave shape of the meniscus that is defined by the Laplace equation. The calculated results are shown to compare with experimental results in Fig. $3 \mathrm{~b}$, and the both agree fairly well, demonstrating the validity of our mechanism. For more details of the model calculation, please refer to Ref. $[62]$.

The concavely curved meniscus against the substrate is thus crucial in the mechanism. In order to confirm further the validity of the mechanism, we attempt to directly observe the shape of the meniscus that is attached to the outermost particle layer away from the substrate. As a model system for the observation, we use a slide glass with the thickness of $1 \mathrm{~mm}$ as a substitute for the particulate film and capture a side view of the behavior of the meniscus shape attached to the bottom-right edge of the slide glass, when the liquid level descends gradually. Because the present matter of interest in the stripe formation is on the micrometer-scale, which is sufficiently large to exhibit bulk-like behavior, the physical properties of water are the same for its contact with both micrometer-scale and millimeter-scale materials. We therefore believe that this observation is surely adequate to test the mechanism. The observation result shows that the meniscus shape indeed starts deforming into a concave shape as the liquid level descends. Furthermore the shape can be well described by the Laplace equation, as shown by the dashed line (Fig. 5b), which strongly supports our proposed mechanism of the stripe pattern formation in which a concavely shaped meniscus plays a crucially important role. This new mode of stripe pattern formation thus enables us to predict the stripe spacing quantitatively, leading to the establishment of a simple and versatile approach to prepare periodic striped microstructures. 


\subsection{Cluster Array Pattern [63]}

In a lower concentration condition below the stripe formation region $\left(<10^{-6} \mathrm{v} / \mathrm{v}\right)$, another periodic structure spontaneously appears through the convective assembly process. The decrease in the particle flux towards the meniscus tip interestingly turns particulate continuous lines into "dotted" ones, as shown in Fig. 6a. The structure is composed of dotted stripes that are aligned at a constant spacing along the parallel to the contact line. Within these dotted lines, the "dot" line up at an almost constant separation (Fig. 6b), and a dot is found out to be a cluster of silica particles (Fig. 6c). This cluster array pattern is then a 3-fold hierarchical structure formed on completely flat substrate via a one-step self-assembly process, without any chemical or physical template. Further decrease in the particle concentration below $10^{-8}$ produces no periodic patterns but random deposition of particles.

Temperatures of 60 and $70{ }^{\circ} \mathrm{C}$ are favorable for cluster array formation, while assembly at lower temperature of $50{ }^{\circ} \mathrm{C}$ results in a rather disordered deposition of clusters due to insufficient convection caused by a lower evaporation rate. This suggests that faster drying favors cluster array formation. In contrast, at a higher temperature of $80^{\circ} \mathrm{C}$, a similar "dotted" structure forms, although the particulate lines are composed of small particle clusters such as dimers, trimmers, and tetramers with small and rather irregular separations, possibly because a faster evaporation rate at the meniscus tip does not allow enough time for the assembled particles to reorganize into cluster aggregates.

The size of the circular cluster linearly increases with the particle concentration (Fig. 7a) simply because a higher concentration brings more particles to the meniscus tip, resulting in larger clusters. The center-to-center separation between clusters, in contrast, shows no clear trend but rather remains constant against the increase in the particle concentration (Fig. 7b). Although we speculate the solvent physical properties govern the separation, the detailed mechanism on the constant separation is not actually clear, which will be investigated in a 
future study. Similar to the cluster-cluster separation, the spacing between dotted lines is also constant (Fig. 7c). However, the spacing distance is narrower by 5-10 $\mu \mathrm{m}$ than that of the striped patterns, which suggests that the formation process of the line spacing in cluster arrays involves a factor that is absent in the stripe formation process.

The direct observation in fact reveals that the formation process of the cluster arrays is quite different from the lateral sliding motion of the meniscus tip in the stripe formation process, as shown in Fig. 4. Particles get assembled at the drying front and fluctuate vigorously along the contact line to form discrete clusters (Fig. 8a). After a while, the entire meniscus tip suddenly retreats in an instantaneous parallel shift (Fig. 8b) until it stabilizes at a new position, which turns out to be rather close to the stick-and-slip motion (Fig. 8c). This difference in the meniscus tip behavior would come from a different morphology of the assembled structure at the drying front. In the case of the cluster array formation, the meniscus is held by discrete clusters of particles, which is contrast to the case of the stripe formation in which a particulate "line" strongly suspends the meniscus. Because the retention ability of the discrete domains to keep the meniscus must be lower than that of the continuous particulate lines, the discrete domains do not allow the meniscus to stretch unlike the case of the particulate lines, but rather they cooperatively release it at a certain limit, resulting in the observed stick-and-slip motion and a narrower spacing between the dotted lines. Hence, the sliding propagation mode along a particulate line produces a wider spacing, whereas the stick-and-slip mode from a dotted line gives rise to a narrower spacing. In this manner, the convective self-assembly process is demonstrated to have the self-regulating characteristics to produce colloidal patterns, whose structure depends on particle concentration and temperature.

\subsection{Grid Pattern [71]}

As described so far, stripe pattern spontaneously forms on a flat substrate under a specific 
concentration condition through convective self-assembly. We then naturally hit on an idea that a grid pattern fabrication is possible by conducting the stripe formation process twice; in the first step, a hydrophilic substrate is immersed in a suspension to produce a stripe pattern on the substrate, and then in the second step, the substrate with the stripe pattern is rotated by $90^{\circ}$ and again immersed in the suspension (Fig. 9a), which we call two-step convective self-assembly. In spite of apparent simplicity and easiness of the idea itself, the grid pattern formation was found out to be difficult because simply immersing the substrate in the second step did not yield the grid pattern but accumulated particles only around the vertical stripes to form raindrop shaped deposits aligned periodically (Fig. 9b, c). The key requirement we determined for the reproducible formation of well-defined grid patterns is plasma treatment before the second immersion in the suspension to clean the surface and recover the hydrophilicity of the substrate which was degraded during the first assembly step due to its exposure to the atmosphere [72]. In fact, performing the second step after the plasma treatment successfully produces a grid pattern as shown in Fig. 9d, e. Interestingly, the width and spacing of the stripes formed in the first step remains almost unchanged after the second step. The second immersion in the suspension does not remove the particles deposited on the substrate, while those in the second step are narrower by $1 \mu \mathrm{m}$ for the width and $3 \mu \mathrm{m}$ for the spacing than the first ones. Another characteristic observed in the grid structure is that the stripes formed in the second step connect smoothly with the first ones without clear boundaries and their width is broadened near the junctions. Furthermore, most of the junctions are monolayers (Fig. 9f), which is contrary to our expectations in which particles deposited in the second step overlap the first stripes at the junctions. Although some junctions are bilayer, the bilayer area is limited to the lower part of the junction.

An important factor to account for the above results is biased convective flows toward the vertical stripes induced by increased solvent evaporation from wet particulate films. The 
biased flows transfer particles preferentially toward the vertical stripes so that these particles are trapped in the gaps at the side edges of the vertical stripes, resulting in smooth connection of the vertical and horizontal stripes without any clear boundaries. Another critical factor is meniscus geometric effect to guide particles along its shape. We have observed that the meniscus is raised by the vertical stripes probably because they possess a hydrophilic porous body that sucks in the solvent when a substrate with the vertical stripes is immersed in a suspension. There is not enough space for particles to pass through the red-colored region in the schematic shown in Fig. 10a, b because the space is confined by the meniscus, whereas enough space exists for the green-colored region. The particles carried by the biased convective flows accordingly cannot enter the red space, but instead align along the contact line to form a monolayer junction, which results in the broadened stripe shape at junctions (Fig. 9e, f). Under a low concentration condition as in Fig. 9d, e, f, the particle deposition in the green region to form multi-layers barely happens because the growing front of the particulate line is separate from the green region, which is depicted in Fig. 10a. According to this mechanism, a higher concentration condition where the growing front is rather close to the green region because of larger particle flux, as shown in Fig. 10b, would result in the formation of domains of bilayers on the junctions. To confirm this, we conducted the grid formation with a higher concentration in the second step. Fig. 10c shows the resultant grid pattern, and for comparison, Fig. 10d shows the stripe pattern produced on a flat substrate under the same concentration as that employed for the second step. Thus, the difference between Figs. 10c and 10d simply originates from the presence of the vertical stripes. In the grid pattern, only the junctions discretely have a bilayer structure with a compass shape, which would reflect the meniscus geometry on the vertical stripe (Fig. 10c). In contrast, the assembly without vertical stripes produces monolayer stripes (Fig. 10d), demonstrating the preferential assembly toward vertical stripes. 
One of the advantages of the convective self-assembly technique is the versatility to arrange various types and sizes of particles. We have actually confirmed the grid pattern formation with small-sized silica particles $(45 \mathrm{~nm})$ and large-sized silica particles $(270 \mathrm{~nm})$. The grid pattern is also produced from particles of different material: silver particles with diameters of 10-15 nm (Fig. 11a). These grid patterns exhibit a high degree of periodicity, similar to the one obtained using $120 \mathrm{~nm}$ silica particles (Fig. 9d). This wide applicability further enables the formation of a hybrid grid pattern where vertical and horizontal stripes are constructed from different types of particles (Fig. 11b), whose anisotropic property would find various technological applications in electronics and sensors. The facile fabrication of well-defined hybrid patterns by our technique exemplifies an advantage over other methods, particularly those that use templates.

\subsection{Active Control over Stripe Pattern Periodicity [73]}

The convective self-assembly with a fairly low concentration produces well-defined stripe pattern in which the spacing between particulate stripes is dependent on the thickness of the stripes. This in turn means that the possible combinations of stripe widths and spacings are naturally limited by the particle size and the number of layers. To overcome the structural limitation in the stripe periodicity, we incorporate a liquid-level manipulation into the convective self-assembly process to control the contact line motion by pumping the colloidal dispersion out of its reservoir by using a programmable syringe pump (Fig. 12a). While the pump is in paused, dispersed particles assemble to form a particulate stripe, and the liquid level falls gradually due to the solvent evaporation. After a certain period (period of particle deposition), the dispersion is pumped out and the liquid level quickly drops (liquid-level drop distance), thereby terminating the particulate stripe growth. Because the rate of the liquid-level drop $(90 \mu \mathrm{m} / \mathrm{s})$ is quick enough to prevent the contact line from being pinned, particles are not deposited on the substrate during the liquid-level drop, resulting in the 
formation of spacing. After the suction, the contact line is pinned again, and the next stripe starts to form. A typical example of resultant stripe pattern is shown in Fig. 12b, in which the sets of stripes and subsequent spacings labeled as $\mathrm{i}$ and ii are fabricated with different combinations of the two operating parameters (period of particle deposition, liquid-level drop distance). A longer deposition period clearly gives wider stripes, while a longer drop distance gives wider spacings, demonstrating the ability to control the periodicity of the stripe patterns. In addition, multilayer stripes can be produced as well as monolayer stripes simply by using dispersions with a high particle concentration and changing the deposition period. The thickness of particulate stripes increases with a particle concentration (Fig. 12c, d, from bilayer to trilayer) and also a deposition period (Fig. 12d, e, from trilayer to four-layer), because a higher concentration and a longer deposition period can transport more particles into the contact line.

In principle, the stripe width and spacing can be increased without limit by increasing the particle concentration, deposition period, and liquid-level drop distance. Although there exists a lower limit to the width and spacing, the liquid-level manipulation can break the threshold minimum encountered in the normal convective self-assembly. The thinnest stripe with close-packed structure we have achieved so far is $\sim 2 \mu \mathrm{m}$ (when $120 \mathrm{~nm}$ silica particles are used) by using a high concentration suspension and a short deposition period. On the other hand, narrowing the spacing is not straightforward; a smaller amount of suction did not produce a narrower spacing than that obtained by the spontaneous cut-off of the meniscus without liquid-level manipulation, because the liquid-level drop has to be large enough to induce the cut-off of the meniscus tip. We thus introduce the liquid-level raise immediately after the suction, which successfully narrows the spacing in a controlled manner.

Extending the technique by combining it with the multi-step convective self-assembly produces network patterns, as shown in Fig. 13 [74]. The resultant grid structure from 
two-step assembly is well-defined and uniform over the whole substrate (Fig. 13a), and three-step assembly with $60^{\circ}$ rotation of a substrate in each step successfully yields a triangle network structure (Fig. 13b). Furthermore, a flexible substrate (polyethylene terephthalate; PET) with relatively large size $(5 \mathrm{~cm} \times 5 \mathrm{~cm})$ can be easily coated with a grid pattern without clear dependence of the structure on substrate position (Fig. 13c-e).

Although the liquid-level manipulation is the same as the substrate withdrawal method in terms of the substrate motion relative to the liquid level, it is easily integrated into the convective self-assembly process just by using an inexpensive syringe pump. Another advantage of the liquid-level manipulation technique, which we found through our experience using a substrate withdrawal approach, is stable operation because the substrate is disconnected from the driving motor of the pump. This allows the fabrication of uniform patterns with fewer structural defects and distortions than are observed for the normal convective self-assembly, and furthermore enables quick and large-scale fabrication. Although similar approaches have been reported to control the meniscus tip motion $[37,39,49,75,76]$, the main advantage of our technique is controlled fabrication on the basis of theoretical models built up for the stripe width and spacing. We believe this technique has potential for various applications because of its versatility and scalability.

\section{Concluding Remarks}

In the present paper, we introduce our recent results on patterned structure formation of colloidal particles by using the convective self-assembly method of vertical deposition type. This technique can produce several patterns including films, stripes, and cluster arrays, which changes depending on the particle concentration, on completely flat substrates without any chemical or physical templating. Particulate uniform films form on substrates fixed in suspensions at high particle concentrations $\left(>10^{-3} \mathrm{v} / \mathrm{v}\right.$ for $120 \mathrm{~nm}$ silica particle), while 
striped patterns spontaneously appear at lower volume fractions of $10^{-4}-10^{-6} \mathrm{v} / \mathrm{v}$. The decrease in the particle concentration slows the growth rate of a particulate film, which leads to the elongation of the meniscus into a concave shape that plays a primary role in the stripe formation mechanism. Grid patterns are produced by conducting the stripe fabrication process twice with $90^{\circ}$ rotation of a substrate after the first assembly. Upon further decrease in the concentration, cluster arrays are formed from extremely dilute suspensions of $10^{-6}-10^{-8} \mathrm{v} / \mathrm{v}$. The transition from the stripe to cluster array occurs when the particle concentration is so small that the particles assembled at the drying front cannot form a continuous line. The morphology of self-assembled structures at the meniscus tip determines the meniscus tip behavior at the moment when the spacing is created: the sliding propagation mode for the stripes and the stick-and-slip mode for the cluster arrays. The convective self-assembly process is thus demonstrated to have an anomalous self-regulating tendency that leads to varying assemblies of microscopic colloidal structures in response to macroscopic experimental parameters. In addition to the self-regulating property, incorporating the liquid-level manipulation protocol enables the active control in the pattern periodicity and the fabrication of stripe patterns with various stripe widths and spacings that are unachievable with the normal convective self-assembly technique. In this way, the convective self-assembly process is a simple, inexpensive, and versatile template-free technique for fabricating patterned structures of particles, and is believed to have the potential for technological applications in variety of fields.

\section{Acknowledgment}

We thank the editorial board of the Society of Powder Technology, Japan for giving the opportunity to publish the present review paper that is a modified and extended version of the original Japanese paper [77]. This work was supported in part by the Grant-in-Aid for Young 
Scientists (A) (No. 23686109) and (B) (No. 20760514) from MEXT, the Global Centers of Excellence (G-COE) Program of the Japan Society for the Promotion of Science (JSPS), the Core-to-Core (CTC) Program (No. 18004) of JSPS, the Nippon Sheet Glass Foundation for Materials Science and Engineering, Hosokawa Powder Technology Foundation, and the Mizuho Foundation for the Promotion of Sciences.

\section{Figure Caption}

Fig. 1 (a) Schematic illustration of the convective self-assembly technique. (b) Magnified schematic around the meniscus tip.

Fig. 2 (a) SEM image of a particulate film of $120 \mathrm{~nm}$ silica particles prepared from a suspension with concentration of $1.3 \times 10^{-3} \mathrm{v} / \mathrm{v}$. White lines are cracks formed during drying. (b) Magnified SEM image of a. (c) SEM image of a stripe patterned film of $120 \mathrm{~nm}$ silica particles prepared from a suspension with concentration of $2.5 \times 10^{-5} \mathrm{v} / \mathrm{v}$. (d) Magnified SEM image of a stripe in c. (e) Optical image of stripes in c. (f) Confocal laser microscope image and height profile of a stripe in c. Black arrow in panels a, c, e, and f indicates the direction of liquid-level drop, i.e., the stripe growth direction. Panels c, $\mathrm{d}$, e and $\mathrm{f}$ of this figure are adapted with permission from Ref. [62]. Copyright 2009 American Chemical Society.

Fig. 3 (a) Stripe width as a function of the particle concentration, shown for two particle sizes. The numbers on the error bars denote the number of layers of stripes. The error bars indicate the standard deviation of the data. (b) Stripe spacing as a function of the stripe thickness, shown for two particle sizes. The dotted lines show the results obtained using the present model, i.e., the calculated stripe spacing as a function of the stripe thickness. 
Fig. 4 Snapshots of the stripe formation process of $270 \mathrm{~nm}$ silica particles at a concentration of $1.0 \times 10^{-5} \mathrm{v} / \mathrm{v}$. Reprinted with permission from Ref. [63]. Copyright 2012 American Chemical Society.

Fig. 5 (a) Concave shape of the meniscus that produces the stripe pattern. (b) Meniscus shape that attaches to the bottom-right edge of a glass slide when the liquid level descends gradually by aspirating the solvent (water) with a syringe. Panel $b$ is adapted with permission from Ref. [62]. Copyright 2009 American Chemical Society.

Fig. 6 (a) Optical image of cluster arrays obtained using $60 \mathrm{~nm}$ gold particles from a suspension of $1.5 \times 10^{-7} \mathrm{v} / \mathrm{v}$. Black arrow indicates the direction of liquid-level drop. $(\mathrm{b}, \mathrm{c})$ Magnified SEM images of cluster arrays in a. Reprinted with permission from Ref. [63]. Copyright 2012 American Chemical Society.

Fig. 7 Dependence of (a) the number of constituent particles in a cluster, (b) the center-to-center separation between clusters, and (c) the spacing on the particle volume fraction at different temperatures. Error bars indicate the standard deviation. Reprinted with permission from Ref. [63]. Copyright 2012 American Chemical Society.

Fig. 8 Snapshots of the cluster array formation process of $100 \mathrm{~nm}$ gold particles at a concentration of $1.0 \times 10^{-7} \mathrm{v} / \mathrm{v}$. Reprinted with permission from Ref. [63]. Copyright 2012 American Chemical Society.

Fig. 9 (a) Schematic illustration of two-step convective self-assembly method for fabrication of grid patterns. (b) Optical image of a resultant structure from the two-step convective 
self-assembly without plasma treatment of the substrate after the first step. (c) Magnified optical image of a raindrop shaped deposit in b. (d) Optical image and (e) SEM image of grid pattern. (f) Topographic image of grid pattern in $\mathrm{d}$. The particle used is $120 \mathrm{~nm}$ silica particle in this figure. Panels a, d, and e of this figure are adapted with permission from Ref. [71]. Copyright 2011 American Chemical Society.

Fig. $10(a, b) 3 D$ schematics of meniscus raised by vertical stripes, demonstrating particle deposition process under conditions of (a) low and (b) high particle concentration. (c) Optical image of grid pattern prepared from suspensions with a concentration of $1.0 \times 10^{-5} \mathrm{v} / \mathrm{v}$ in the first step and $2.0 \times 10^{-5} \mathrm{v} / \mathrm{v}$ in the second step. (d) Optical image of stripe pattern formed without vertical stripes at the concentration of $2.0 \times 10^{-5} \mathrm{v} / \mathrm{v}$. The particle used is $120 \mathrm{~nm}$ silica particle in this figure. Black arrow indicates the direction of the liquid-level drop. Reprinted with permission from Ref. [71]. Copyright 2011 American Chemical Society.

Fig. 11 (a) Optical image of grid pattern using a suspension of 10-15 nm silver particles at $5.0 \times 10^{-6} \mathrm{v} / \mathrm{v}$ both in the first and second steps. (b) Optical image of silica-silver hybrid grid pattern obtained in first step using $120 \mathrm{~nm}$ silica particles at $1.0 \times 10^{-5} \mathrm{v} / \mathrm{v}$ and second step using $10-15 \mathrm{~nm}$ silver particles at $5.0 \times 10^{-6} \mathrm{v} / \mathrm{v}$. Reprinted with permission from Ref. [71]. Copyright 2011 American Chemical Society.

Fig. 12 (a) Schematic illustration of the convective self-assembly with liquid-level manipulation technique. (b) Optical image of a typical stripe pattern obtained from a $120 \mathrm{~nm}$ silica particle suspension of $3.0 \times 10^{-5} \mathrm{v} / \mathrm{v}$ by convective self-assembly with liquid-level manipulation. The sets of particulate stripes and subsequent spacings labeled as i and ii are fabricated with different combinations of the two operating parameters, (deposition period, 
liquid-level drop distance $)=(60 \mathrm{~s}, 40 \mu \mathrm{m})$ and $(120 \mathrm{~s}, 70 \mu \mathrm{m})$. (c-e) Optical images of multilayer stripe patterns. (c) Bilayer, $5.0 \times 10^{-5} \mathrm{v} / \mathrm{v},(60 \mathrm{~s}, 70 \mu \mathrm{m})$. (d) Trilayer, $1.0 \times 10^{-4} \mathrm{v} / \mathrm{v}$, $(120 \mathrm{~s}, 70 \mu \mathrm{m})$. (e) Four-layer, $1.0 \times 10^{-4} \mathrm{v} / \mathrm{v},(180 \mathrm{~s}, 70 \mu \mathrm{m})$. Reprinted with permission from Ref. [73]. Copyright 2012 American Chemical Society.

Fig. 13 Optical images of (a) grid pattern and (b) triangle network from a $20 \mathrm{~nm}$ gold particle suspension at $1.0 \times 10^{-4} \mathrm{v} / \mathrm{v}$ by combining the convective self-assembly with liquid-level manipulation with the two/three-step convective self-assembly. (c) Photograph and (d, e) optical micrographs of a large-sized grid network pattern of silver nanoparticles that was fabricated on the $5 \mathrm{~cm} \times 5 \mathrm{~cm}$ PET substrate from a 10-15 $\mathrm{nm}$ silver particle suspension of $1.0 \times 10^{-5} \mathrm{v} / \mathrm{v}$. Panels a and $\mathrm{b}$ of this figure are from Ref. [74], and c, d, and e are adapted with permission from Ref. [73]. Copyright 2012 American Chemical Society.

\section{References}

[1] B.D. Gates, Q. Xu, M. Stewart, D. Ryan, C.G. Willson, G.M. Whitesides, New Approaches to Nanofabrication: Molding, Printing, and Other Techniques, Chem. Rev., 105 (2005) 1171-1196.

[2] P. Innocenzi, T. Kidchob, P. Falcaro, M. Takahashi, Patterning Techniques for Mesostructured Films, Chem. Mater., 20 (2008) 607-614.

[3] S. Tawfick, M. De Volder, D. Copic, S.J. Park, C.R. Oliver, E.S. Polsen, M.J. Roberts, A.J. Hart, Engineering of Micro- and Nanostructured Surfaces with Anisotropic Geometries and Properties, Adv. Mater., 24 (2012) 1628-1674.

[4] M. Zhu, G. Baffou, N. Meyerbröker, J. Polleux, Micropatterning Thermoplasmonic Gold Nanoarrays to Manipulate Cell Adhesion, ACS Nano, 6 (2012) 7227-7233.

[5] M. Layani, M. Gruchko, O. Milo, I. Balberg, D. Azulay, S. Magdassi, Transparent Conductive Coatings by Printing Coffee Ring Arrays Obtained at Room Temperature, ACS Nano, 3 (2009) 3537-3542.

[6] K. Higashitani, C.E. McNamee, M. Nakayama, Formation of Large-Scale Flexible Transparent Conductive Films Using Evaporative Migration Characteristics of Au Nanoparticles, Langmuir, 27 (2011) 2080-2083. 
[7] J.A. Fan, K. Bao, L. Sun, J. Bao, V.N. Manoharan, P. Nordlander, F. Capasso, Plasmonic Mode Engineering with Templated Self-Assembled Nanoclusters, Nano Lett., (2012).

[8] X.C. Jiang, Q.H. Zeng, C.Y. Chen, A.B. Yu, Self-Assembly of Particles: Some Thoughts and Comments, J. Mater. Chem., 21 (2011) 16797-16805.

[9] N.B. Crane, O. Onen, J. Carballo, Q. Ni, R. Guldiken, Fluidic Assembly at the Microscale: Progress and Prospects, Microfluid. Nanofluid., 14 (2012) 383-419.

[10] W. Han, Z. Lin, Learning from "Coffee Rings": Ordered Structures Enabled by Controlled Evaporative Self-Assembly, Angew. Chem., Int. Ed., 51 (2012) 1534-1546.

[11] Y. Yin, Y. Lu, B. Gates, Y. Xia, Template-Assisted Self-Assembly: A Practical Route to Complex Aggregates of Monodispersed Colloids with Well-Defined Sizes, Shapes, and Structures, J. Am. Chem. Soc., 123 (2001) 8718-8729.

[12] D. Xia, A. Biswas, D. Li, S.R.J. Brueck, Directed Self-Assembly of Silica Nanoparticles into Nanometer-Scale Patterned Surfaces Using Spin-Coating, Adv. Mater., 16 (2004) 1427-1432.

[13] N.N. Khanh, K.B. Yoon, Facile Organization of Colloidal Particles into Large, Perfect One- and Two-Dimensional Arrays by Dry Manual Assembly on Patterned Substrates, J. Am. Chem. Soc., 131 (2009) 14228-14230.

[14] Y. Masuda, T. Itoh, K. Koumoto, Self-Assembly Patterning of Silica Colloidal Crystals, Langmuir, 21 (2005) 4478-4481.

[15] Y.-J. Choi, T.-J.M. Luo, Self-Assembly of Silver-Aminosilica Nanocomposites through Silver Nanoparticle Fusion on Hydrophobic Surfaces, ACS Appl. Mater. Interfaces, 1 (2009) 2778-2784.

[16] S. Yasumatsu, H. Ishizuka, K. Nakaso, P.O. Babatunde, J. Fukai, Self-Organization of Polymer Films Using Single and Mixed Solvents on Chemically Patterned Surfaces, J. Chem. Eng. Japan, 45 (2012) 265-271.

[17] H. Celio, E. Barton, K.J. Stevenson, Patterned Assembly of Colloidal Particles by Confined Dewetting Lithography, Langmuir, 22 (2006) 11426-11435.

[18] D.J. Harris, H. Hu, J.C. Conrad, J.A. Lewis, Patterning Colloidal Films via Evaporative Lithography, Phys. Rev. Lett., 98 (2007) 148301.

[19] J.X. Huang, F. Kim, A.R. Tao, S. Connor, P.D. Yang, Spontaneous Formation of Nanoparticle Stripe Patterns through Dewetting, Nat. Mater., 4 (2005) 896-900.

[20] H. Yabu, M. Shimomura, Preparation of Self-Organized Mesoscale Polymer Patterns on a Solid Substrate: Continuous Pattern Formation from a Receding Meniscus, Adv. Funct. Mater., 15 (2005) 575-581. 
[21] R. van Hameren, P. Schön, A.M. van Buul, J. Hoogboom, S.V. Lazarenko, J.W. Gerritsen, H. Engelkamp, P.C.M. Christianen, H.A. Heus, J.C. Maan, T. Rasing, S. Speller, A.E. Rowan, J.A.A.W. Elemans, R.J.M. Nolte, Macroscopic Hierarchical Surface Patterning of Porphyrin Trimers via Self-Assembly and Dewetting, Science, 314 (2006) 1433-1436.

[22] O. Karthaus, L. Gråsjö, N. Maruyama, M. Shimomura, Formation of Ordered Mesoscopic Polymer Arrays by Dewetting, Chaos, 9 (1999) 308-314.

[23] N.J. Suematsu, Y. Ogawa, Y. Yamamoto, T. Yamaguchi, Dewetting Self-Assembly of Nanoparticles into Hexagonal Array of Nanorings, J. Colloid Interface Sci., 310 (2007) 648-652.

[24] Y. Cai, B.-m. Zhang Newby, Marangoni Flow-Induced Self-Assembly of Hexagonal and Stripelike Nanoparticle Patterns, J. Am. Chem. Soc., 130 (2008) 6076-6077.

[25] C.A. Rezende, L.-T. Lee, F. Galembeck, Liquid Templating for Nanoparticle Organization into Complex Patterns, Langmuir, 23 (2007) 2824-2828.

[26] A.M. Cazabat, F. Heslot, S.M. Troian, P. Carles, Fingering Instability of Thin Spreading Films Driven by Temperature Gradients, Nature, 346 (1990) 824-826.

[27] S. Schiaffino, A.A. Sonin, Formation and Stability of Liquid and Molten Beads on a Solid Surface, J. Fluid Mech., 343 (1997) 95-110.

[28] E. Adachi, A.S. Dimitrov, K. Nagayama, Stripe Patterns Formed on a Glass Surface During Droplet Evaporation, Langmuir, 11 (1995) 1057-1060.

[29] R.D. Deegan, Pattern Formation in Drying Drops, Phys. Rev. E, 61 (2000) 475-485.

[30] L. Shmuylovich, A.Q. Shen, H.A. Stone, Surface Morphology of Drying Latex Films: Multiple Ring Formation, Langmuir, 18 (2002) 3441-3445.

[31] T. Okubo, S. Okuda, H. Kimura, Dissipative Structures Formed in the Course of Drying the Colloidal Crystals of Silica Spheres on a Cover Glass, Colloid Polym. Sci., 280 (2002) 454-460.

[32] R.D. Deegan, O. Bakajin, T.F. Dupont, G. Huber, S.R. Nagel, T.A. Witten, Capillary Flow as the Cause of Ring Stains from Dried Liquid Drops, Nature, 389 (1997) 827-829.

[33] Y. Masuda, T. Itoh, M. Itoh, K. Koumoto, Self-Assembly Patterning of Colloidal Crystals Constructed from Opal Structure or Nacl Structure, Langmuir, 20 (2004) 5588-5592.

[34] J.X. Huang, A.R. Tao, S. Connor, R. He, P. Yang, A General Method for Assembling Single Colloidal Particle Lines, Nano Lett., 6 (2006) 524-529.

[35] M. Ghosh, F. Fan, K.J. Stebe, Spontaneous Pattern Formation by Dip Coating of Colloidal Suspensions on 
Homogeneous Surfaces, Langmuir, 23 (2007) 2180-2183.

[36] J. Xu, J.F. Xia, Z.Q. Lin, Evaporation-Induced Self-Assembly of Nanoparticles from a Sphere-on-Flat Geometry, Angew. Chem. Int. Ed., 46 (2007) 1860-1863.

[37] H.S. Kim, C.H. Lee, P.K. Sudeep, T. Emrick, A.J. Crosby, Nanoparticle Stripes, Grids, and Ribbons Produced by Flow Coating, Adv. Mater., 22 (2010) 4600-4604.

[38] W. Han, M. Byun, Z. Lin, Assembling and Positioning Latex Nanoparticles via Controlled Evaporative Self-Assembly, J. Mater. Chem., 21 (2011) 16968-16972.

[39] C. Farcau, N.M. Sangeetha, H. Moreira, B. Viallet, J. Grisolia, D. Ciuculescu-Pradines, L. Ressier, High-Sensitivity Strain Gauge Based on a Single Wire of Gold Nanoparticles Fabricated by Stop-and-Go Convective Self-Assembly, ACS Nano, 5 (2011) 7137-7143.

[40] A.S. Dimitrov, K. Nagayama, Continuous Convective Assembling of Fine Particles into Two-Dimensional Arrays on Solid Surfaces, Langmuir, 12 (1996) 1303-1311.

[41] O. Giraldo, J.P. Durand, H. Ramanan, K. Laubernds, S.L. Suib, M. Tsapatsis, S.L. Brock, M. Marquez, Dynamic Organization of Inorganic Nanoparticles into Periodic Micrometer-Scale Patterns, Angew. Chem. Int. Ed., 42 (2003) 2905-2909.

[42] N.D. Denkov, O.D. Velev, P.A. Kralchevsky, I.B. Ivanov, H. Yoshimura, K. Nagayama, Two-Dimensional Crystallization, Nature, 361 (1993) 26.

[43] P. Jiang, J.F. Bertone, K.S. Hwang, V.L. Colvin, Single-Crystal Colloidal Multilayers of Controlled Thickness, Chem. Mater., 11 (1999) 2132-2140.

[44] D.D. Brewer, T. Shibuta, L. Francis, S. Kumar, M. Tsapatsis, Coating Process Regimes in Particulate Film Production by Forced-Convection-Assisted Drag-Out, Langmuir, (2011) null-null.

[45] M.P. Pileni (Ed.), Nanocrystals Forming Mesoscopic Structures, Wiley-VCH, 2006.

[46] J.M. Meijer, F. Hagemans, L. Rossi, D.V. Byelov, S. Castillo, A. Snigirev, I. Snigireva, A.P. Philipse, A.V. Petukhov, Self-Assembly of Colloidal Cubes via Vertical Deposition, Langmuir, (2012).

[47] N. Yanai, S. Granick, Directional Self-Assembly of a Colloidal Metal-Organic Framework, Angew. Chem., Int. Ed., (2012).

[48] J.A. Lee, L. Meng, D.J. Norris, L.E. Scriven, M. Tsapatsis, Colloidal Crystal Layers of Hexagonal Nanoplates by Convective Assembly, Langmuir, 22 (2006) 5217-5219.

[49] J.X. Huang, R. Fan, S. Connor, P. Yang, One-Step Patterning of Aligned Nanowire Arrays by Programmed Dip Coating, Angew. Chem. Int. Ed., 46 (2007) 2414-2417. 
[50] C. Zhang, X. Zhang, X. Zhang, X. Fan, J. Jie, J.C. Chang, C.-S. Lee, W. Zhang, S.-T. Lee, Facile One-Step Growth and Patterning of Aligned Squaraine Nanowires via Evaporation-Induced Self-Assembly, Adv. Mater., 20 (2008) 1716-1720.

[51] H. Zeng, K. Kristiansen, P. Wang, J. Bergli, J. Israelachvili, Surface-Induced Patterns from Evaporating Droplets of Aqueous Carbon Nanotube Dispersions, Langmuir, 27 (2011) 7163-7167.

[52] L. Xiao, J. Wei, Y. Gao, D. Yang, H. Li, Formation of Gradient Multi-Walled Carbon Nanotube Stripe Patterns by Using Evaporation-Induced Self-Assembly, ACS Appl. Mater. Interfaces, (2012).

[53] X. Zhang, Y. Wang, S. Watanabe, M.H. Uddin, D. Li, Evaporation-Induced Flattening and Self-Assembly of Chemically Converted Graphene on a Solid Surface, Soft Matter, 7 (2011) 8745-8748.

[54] Y. Wang, Y. Song, S. Watanabe, S. Zhang, D. Li, X. Zhang, Stitching Chemically Converted Graphene on Solid Surfaces by Solvent Evaporation, ACS Appl. Mater. Interfaces, (2012).

[55] Y.A. Vlasov, X.-Z. Bo, J.C. Sturm, D.J. Norris, On-Chip Natural Assembly of Silicon Photonic Bandgap Crystals, Nature, 414 (2001) 289-293.

[56] S.M. Yang, H. Míguez, G.A. Ozin, Opal Circuits of Light-Planarized Microphotonic Crystal Chips, Adv. Funct. Mater., 12 (2002) 425-431.

[57] S. Wong, V. Kitaev, G.A. Ozin, Colloidal Crystal Films: Advances in Universality and Perfection, J. Am. Chem. Soc., 125 (2003) 15589-15598.

[58] A.D. Ormonde, E.C.M. Hicks, J. Castillo, R.P. Van Duyne, Nanosphere Lithography: Fabrication of Large-Area Ag Nanoparticle Arrays by Convective Self-Assembly and Their Characterization by Scanning Uv-Visible Extinction Spectroscopy, Langmuir, 20 (2004) 6927-6931.

[59] B.G. Prevo, O.D. Velev, Controlled, Rapid Deposition of Structured Coatings from Micro- and Nanoparticle Suspensions, Langmuir, 20 (2004) 2099-2107.

[60] K. Chen, S.V. Stoianov, J. Bangerter, H.D. Robinson, Restricted Meniscus Convective Self-Assembly, J. Colloid Interface Sci., 344 (2010) 315-320.

[61] W. Kern, D.A. Puotinen, Cleaning Solutions Based on Hydrogen Peroxide for Use in Silicon Semiconductor Technology, RCA Rev., 31 (1970) 187-206.

[62] S. Watanabe, K. Inukai, S. Mizuta, M.T. Miyahara, Mechanism for Stripe Pattern Formation on Hydrophilic Surfaces by Using Convective Self-Assembly, Langmuir, 25 (2009) 7287-7295.

[63] S. Watanabe, Y. Mino, Y. Ichikawa, M.T. Miyahara, Spontaneous Formation of Cluster Array of Gold Particles by Convective Self-Assembly, Langmuir, 28 (2012) 12982-12988. 
[64] W.P. Lee, A.F. Routh, Why Do Drying Films Crack?, Langmuir, 20 (2004) 9885-9888.

[65] S. Kitsunezaki, Crack Growth in Drying Paste, Adv. Powder Technol., 22 (2011) 311-318.

[66] L.K. Wang, X.S. Zhao, Fabrication of Crack-Free Colloidal Crystals Using a Modified Vertical Deposition Method, J. Phys. Chem. C, 111 (2007) 8538-8542.

[67] J.H. Prosser, T. Brugarolas, S. Lee, A.J. Nolte, D. Lee, Avoiding Cracks in Nanoparticle Films, Nano Lett., (2012).

[68] C.D. Dushkin, K. Nagayama, T. Miwa, P.A. Kralchevsky, Colored Multilayers from Transparent Submicrometer Spheres, Langmuir, 9 (1993) 3695-3701.

[69] H. Bodiguel, F. Doumenc, B. Guerrier, Stick-Slip Patterning at Low Capillary Numbers for an Evaporating Colloidal Suspension, Langmuir, 26 (2010) 10758-10763.

[70] L. Frastia, A.J. Archer, U. Thiele, Dynamical Model for the Formation of Patterned Deposits at Receding Contact Lines, Phys. Rev. Lett., 106 (2011).

[71] Y. Mino, S. Watanabe, M.T. Miyahara, Fabrication of Colloidal Grid Network by Two-Step Convective Self-Assembly, Langmuir, 27 (2011) 5290-5295.

[72] K.F. Baughman, R.M. Maier, T.A. Norris, B.M. Beam, A. Mudalige, J.E. Pemberton, J.E. Curry, Evaporative Deposition Patterns of Bacteria from a Sessile Drop: Effect of Changes in Surface Wettability Due to Exposure to a Laboratory Atmosphere, Langmuir, 26 (2010) 7293-7298.

[73] Y. Mino, S. Watanabe, M.T. Miyahara, Colloidal Stripe Pattern with Controlled Periodicity by Convective Self-Assembly with Liquid-Level Manipulation, ACS Appl. Mater. Interfaces, 4 (2012) 3184-3190.

[74] T. Hanafusa, Y. Mino, S. Watanabe, M.T. Miyahara, Controlling Self-Assembled Structure of Au Nanoparticles by Convective Self-Assembly with Liquid-Level Manipulation, J. Soc. Powder Technol., Japan, 49 (2012) 356-361.

[75] J.J. Diao, J. Sun, J.B. Hutchison, M.E. Reeves, Self Assembled Nanoparticle Wires by Discontinuous Vertical Colloidal Deposition, Appl. Phys. Lett., 87 (2005) 103113.

[76] R. Sakamoto, Y. Hataguchi, R. Kimura, K. Tsuchiya, Y. Mori, Stripe and Network Formation of Particle Arrays Fabricated by Convective Self-Assembly, Chem. Lett., 41 (2012) 1207-1209.

[77] S. Watanabe, Colloidal Pattern Formation and Its Morphology Control by Convective Self-Assembly, J. Soc. Powder Technol., Japan, 48 (2011) 312-318. 
(a)

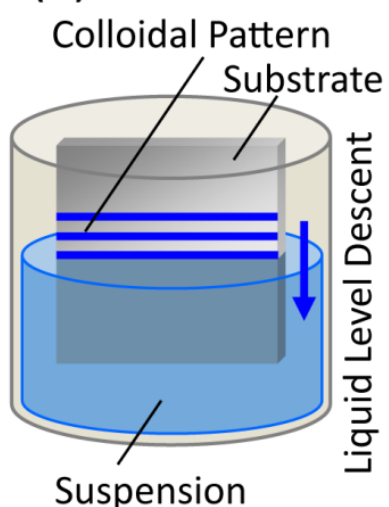

(b)

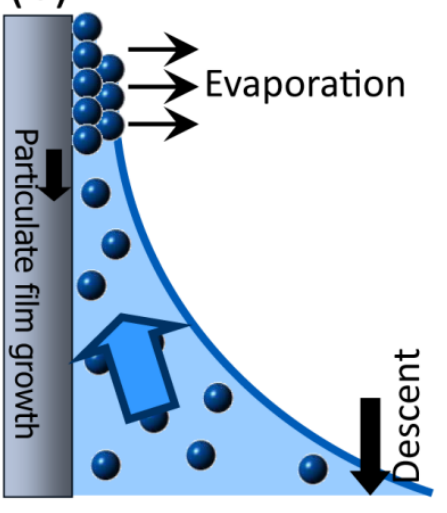



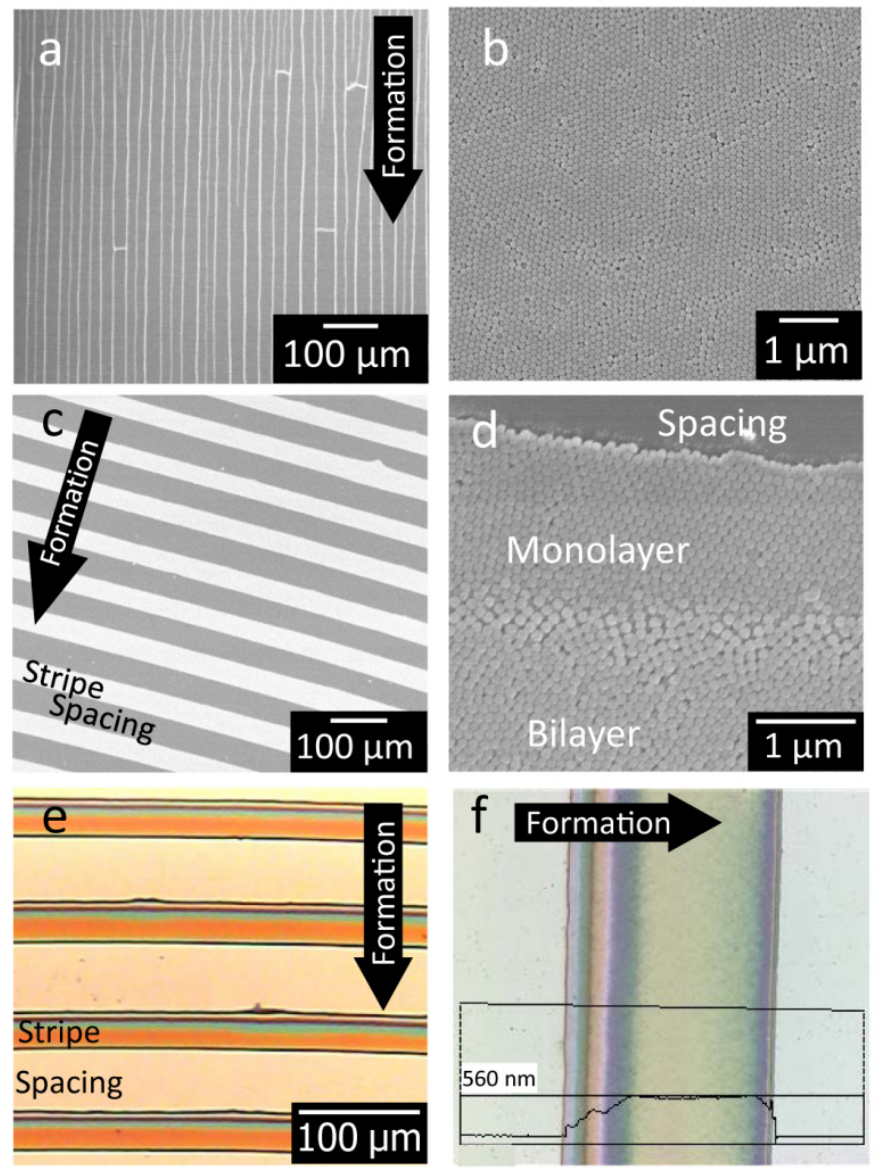

Fig. 2 Watanabe \& Miyahara 
(a)
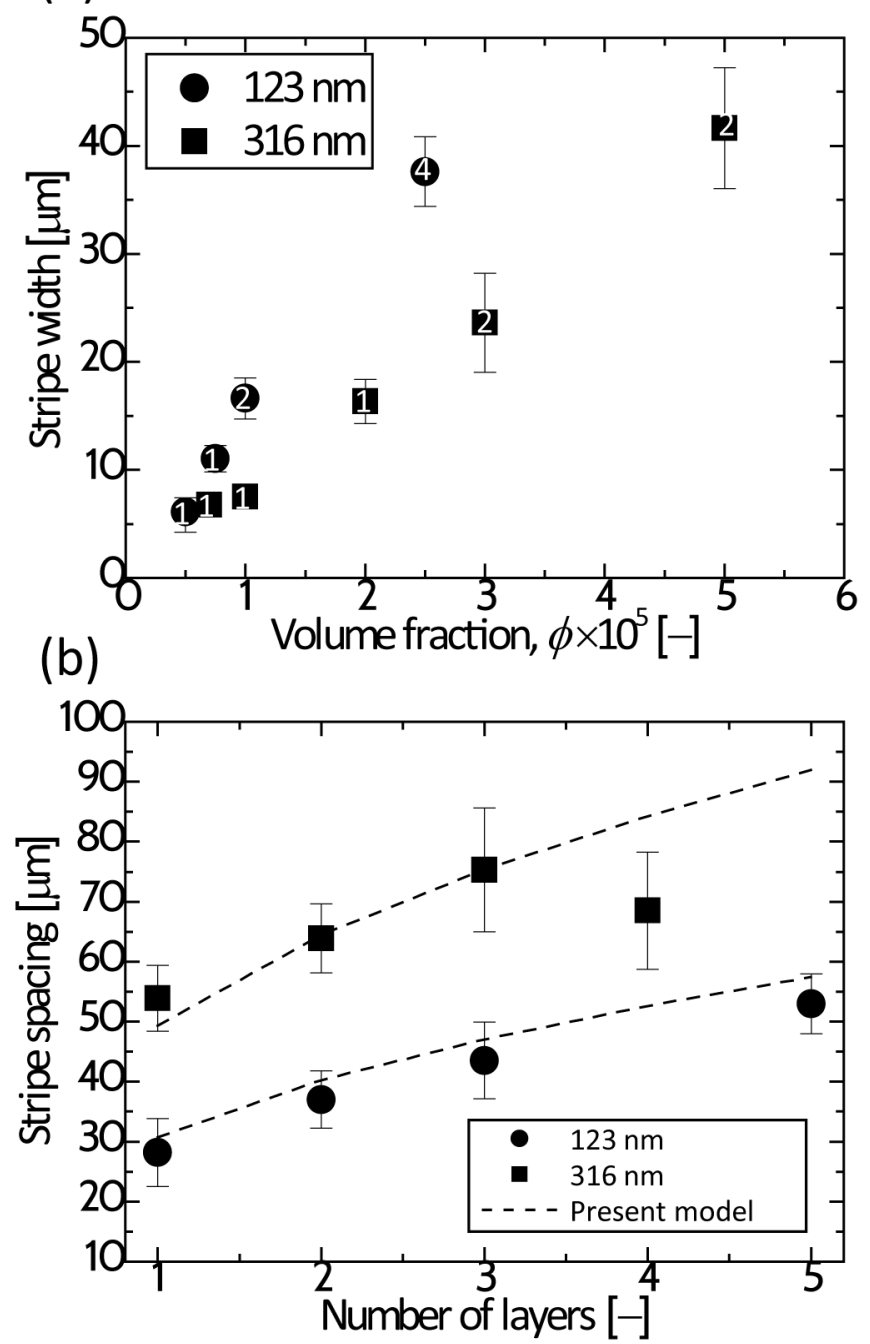

Fig. 3 Watanabe \& Miyahara 


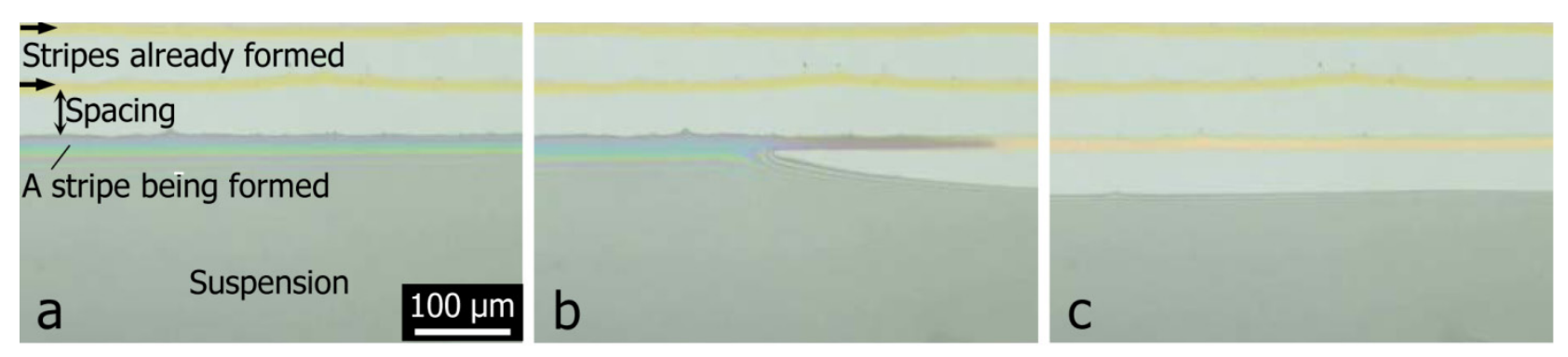

Fig. 4 Watanabe \& Miyahara 
(a)

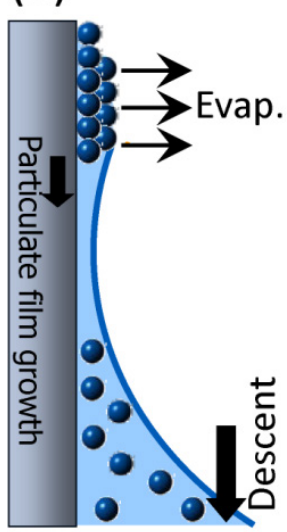

(b)

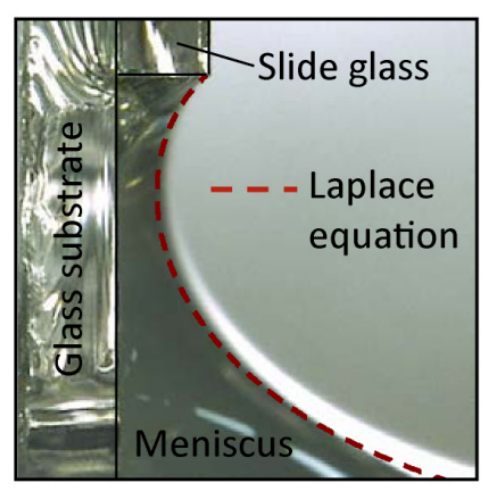

Fig. 5 Watanabe \& Miyahara 


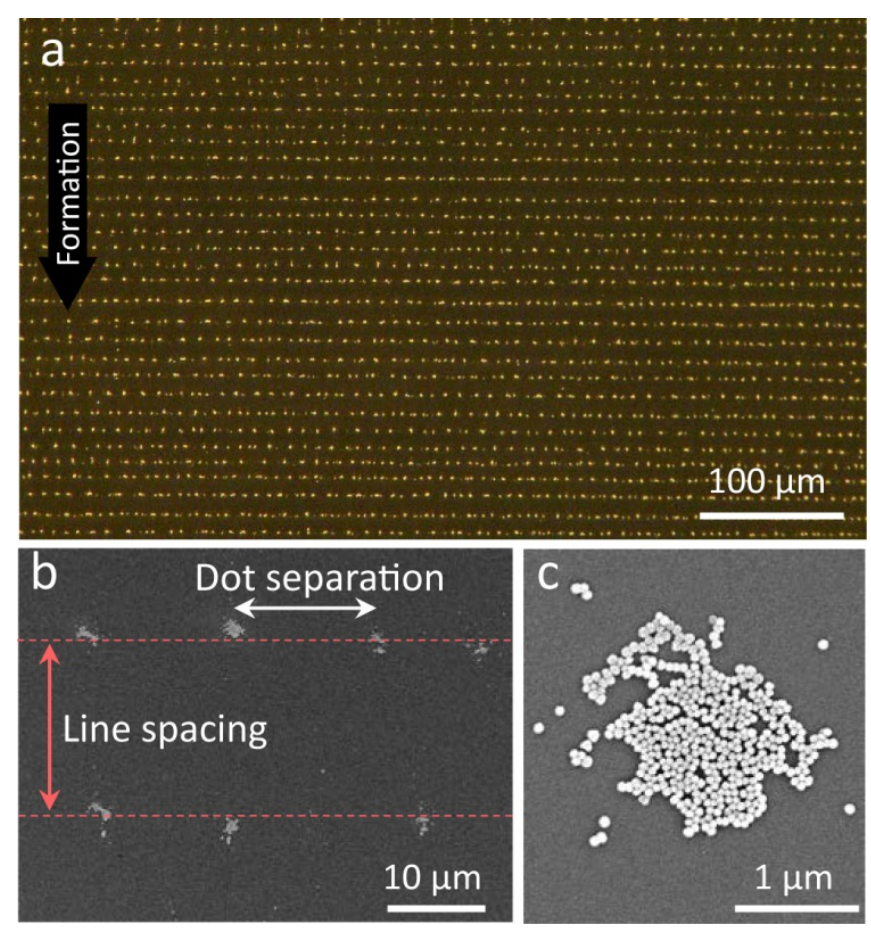

Fig. 6 Watanabe \& Miyahara 
(a)

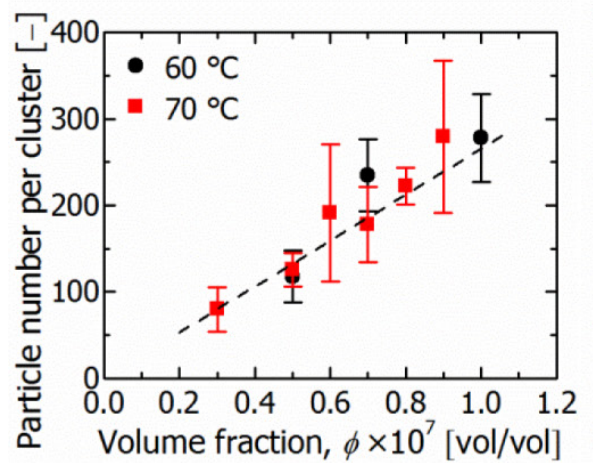

(b)

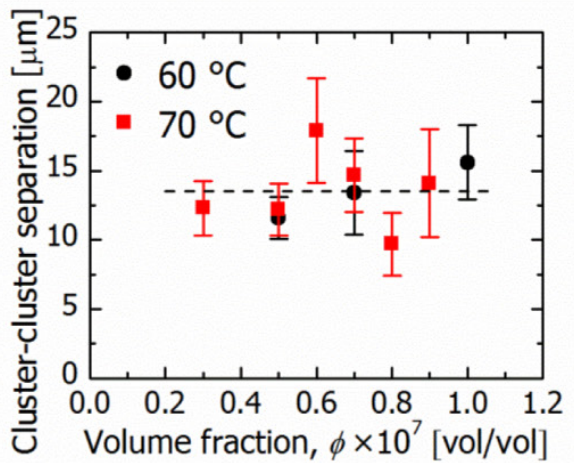

(c)

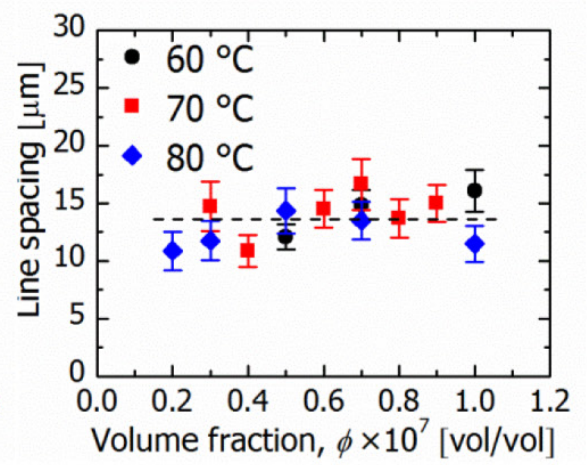




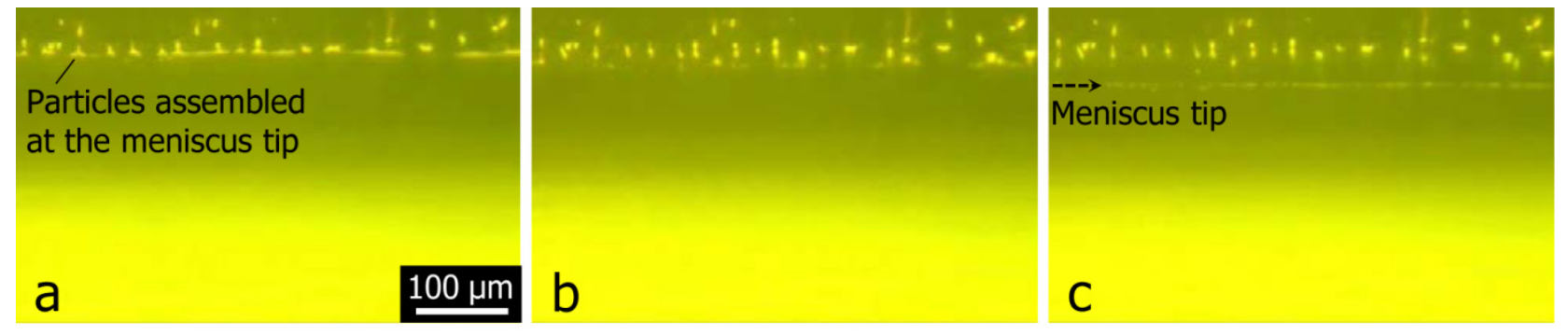

Fig. 8 Watanabe \& Miyahara 

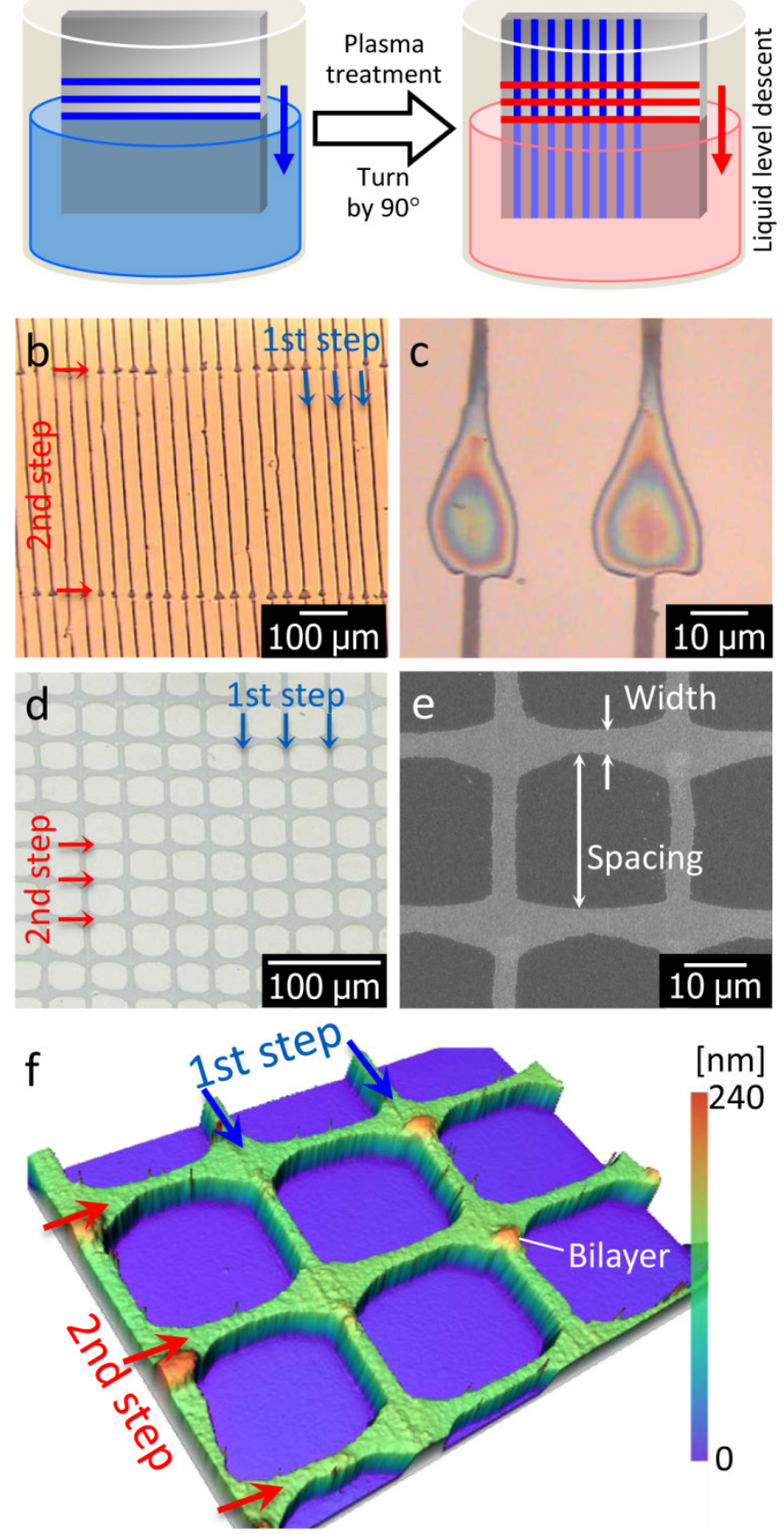

Fig. 9 Watanabe \& Miyahara 

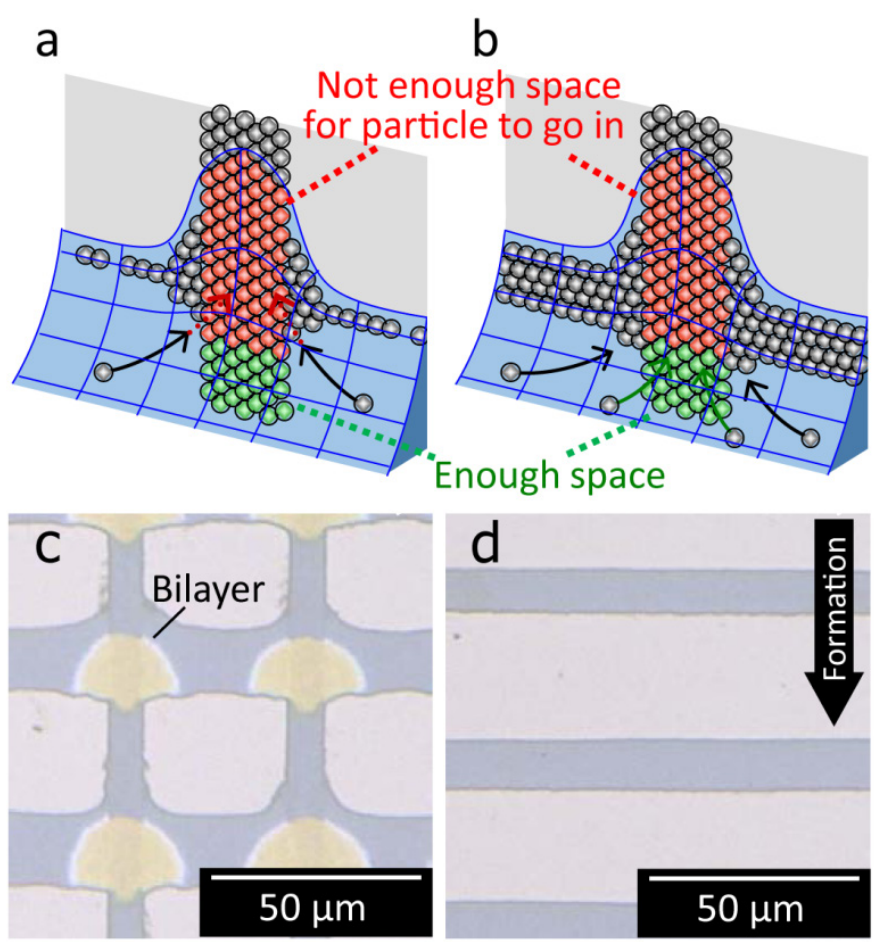

Fig. 10 Watanabe \& Miyahara 


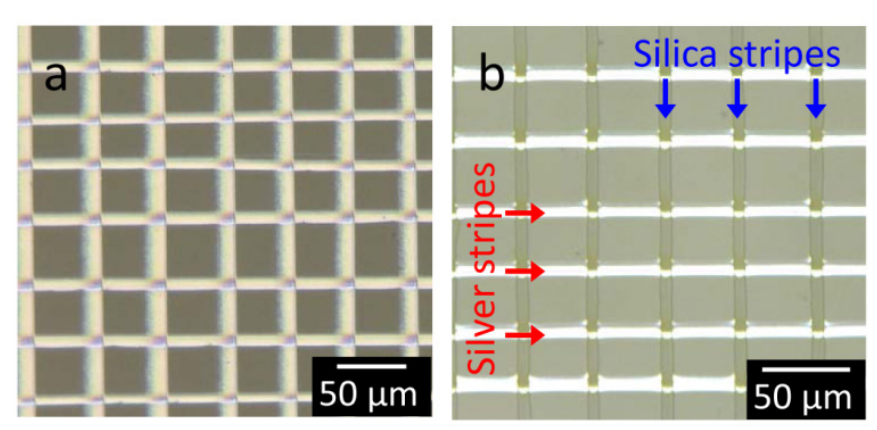

Fig. 11 Watanabe \& Miyahara 
$a$

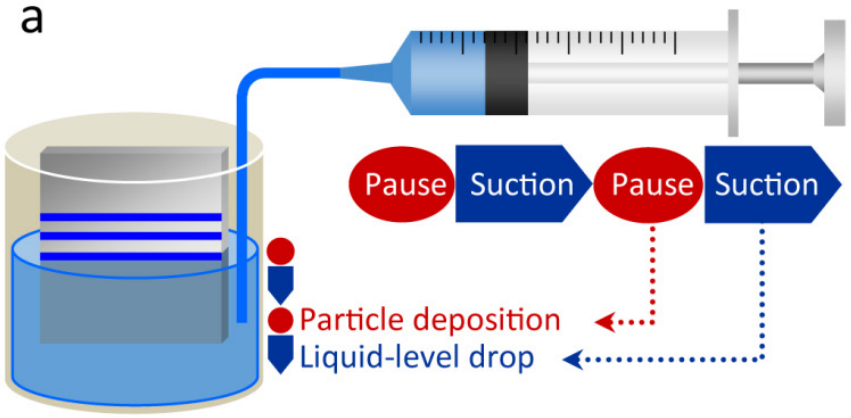

b
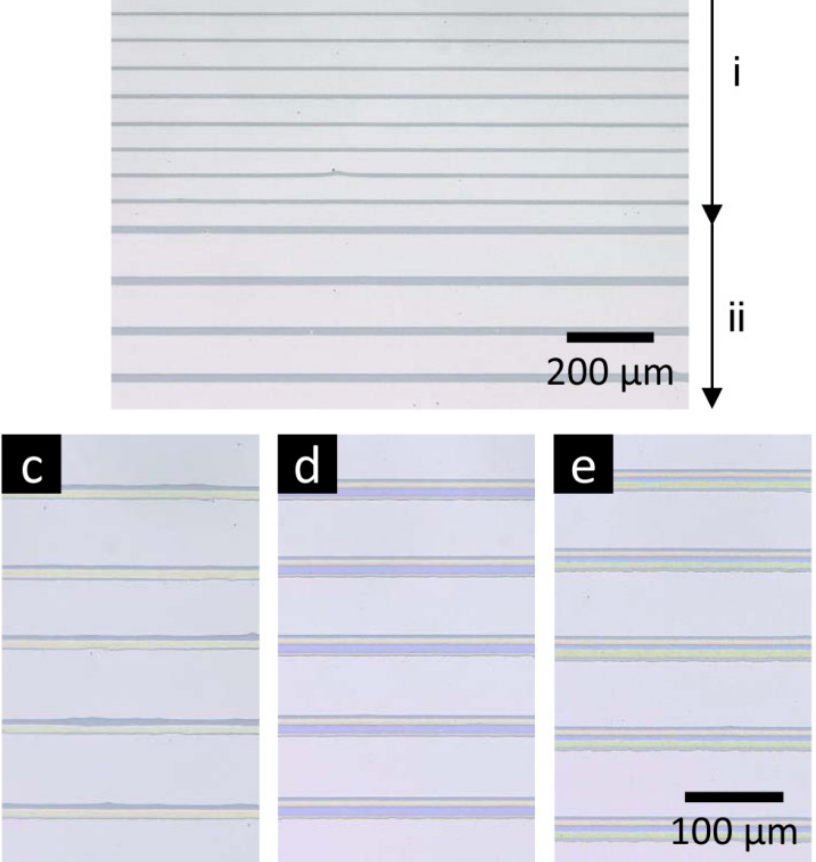

Fig. 12 Watanabe \& Miyahara 

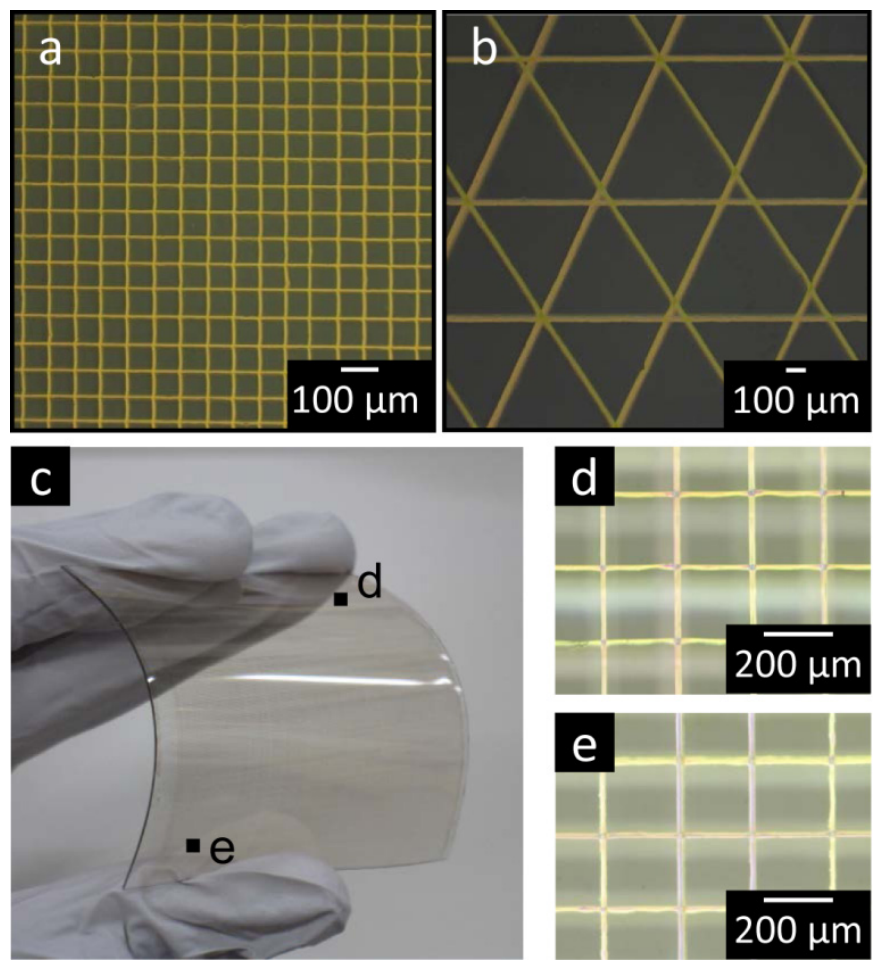

Fig. 13 Watanabe \& Miyahara 


\section{Template-Free Fabrication}

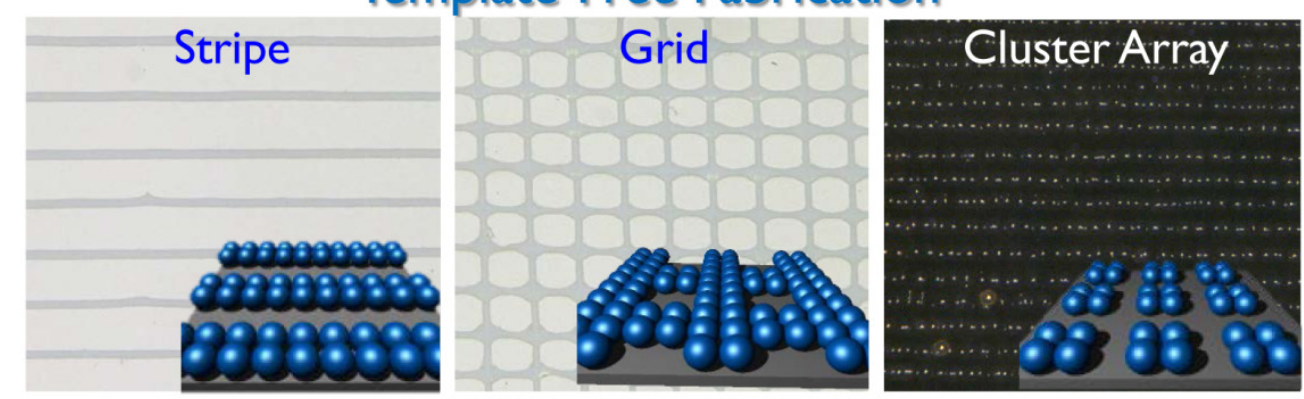

Graphical Abstract, Watanabe \& Miyahara 